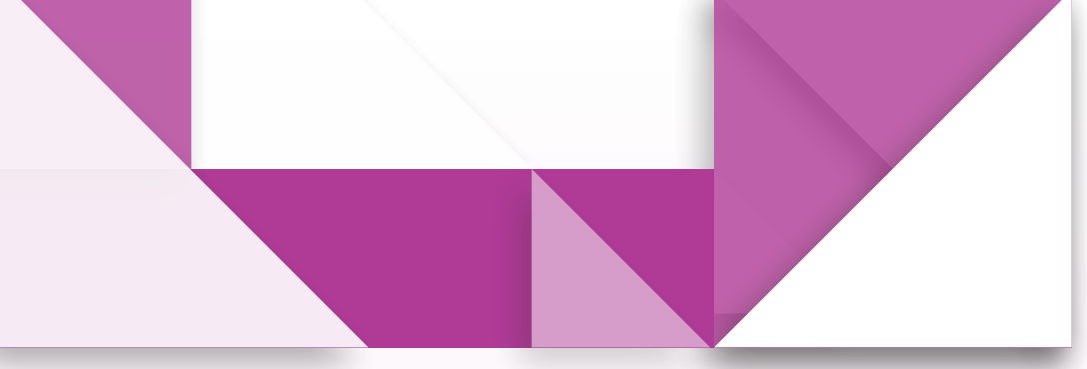

\title{
1) El aula invertida y la $>$ construcción de conocimiento en matemáticas. El caso de las aplicaciones de la derivada
}

- The Flipped Classroom and the Construction of Knowledge in Mathematics. The Case of Derivative Applications

- A sala de aula invertida e a construção do conhecimento em matemática. $\bigcirc$ caso de aplicações derivadas

\section{Resumen}

Este artículo de investigación describe y analiza la implementación del "aula invertida" en el desarrollo de dos clases de cálculo diferencial, en primer semestre universitario, abordando el concepto matemático de derivada desde su aplicación en problemas de razones de cambio, velocidades, máximos y mínimos. El análisis se realiza a partir de grabaciones de los episodios de clase y de la interacción en redes sociales entre los estudiantes; se describen los aspectos positivos y negativos encontrados y su relación con el éxito académico que alcanzaron los estudiantes. Lo anterior permitió revelar la necesidad de un desarrollo más profundo de los fundamentos de esta metodología en lo correspondiente al proceso de aprendizaje del objeto matemático derivada por parte de los estudiantes.

Palabras clave

aula invertida; aprendizaje de la derivada; aprendizaje en el nivel universitario; uso de las TIC en el aprendizaje
Cristian Camilo Fúneme Mateus ${ }^{1}$

Estudiante de la Maestría en Educación Matemática. Universidad Pedagógica y Tecnológica de Colombia. Tunja, Boyacá, Colombia. cristian.funeme@uptc.edu.co Orcid: 0000-0002-9158-427X 


\begin{abstract}
This research paper describes and analyzes the implementation of the "flipped classroom" in two first-semester differential calculus classes at a university, approaching the mathematical concept of derivatives from their application in rate-of-change, speed, maximums and minimums problems. The analysis is made based on class recordings and on the students' interactions in social networks; the positive and negative aspects found are described, as well as their relationship with the academic success achieved by the students. This revealed the need for further development of the fundamentals of this methodology as regards the students' process of learning about derivatives.
\end{abstract}

Keywords

flipped classroom; derivative learning; higher education learning; use of ICT in learning

\title{
Resumo
}

Este artigo de pesquisa descreve e analisa a implementação da "sala de aula invertida" no desenvolvimento de duas aulas de cálculo diferencial, no primeiro semestre, abordando o conceito matemático de derivada desde sua aplicação em problemas de proporções, velocidades, máximos e mínimos. A análise é feita a partir de gravações das aulas e da interação nas redes sociais entre os alunos; descrevemos os aspectos positivos e negativos encontrados e sua relação com o sucesso acadêmico alcançado pelos alunos. Isso permitiu revelar a necessidade de um desenvolvimento mais profundo dos fundamentos dessa metodologia no processo de aprendizagem do objeto matemático derivada pelos alunos.

Palavras-chave

sala de aula invertida; aprendizagem derivada; aprendizagem no nível universitário; uso das TIC na aprendizagem 


\section{Introducción}

La didáctica de las matemáticas tiene como principal preocupación el estudio de los factores que condicionan los procesos de enseñanza y aprendizaje de las matemáticas y el cómo mejorarlos (Brousseau, 1986; Godino, Batanero y Font, 2007), siendo de vital importancia la incorporación del docente como investigador para lograr un real impacto en las instituciones educativas de lo alcanzado teóricamente.

Entre los conceptos matemáticos para los cuales se ha encontrado especial dificultad en el desarrollo del proceso de aprendizaje, se halla la derivada. Este hecho se ha evidenciado en diferentes investigaciones, como por ejemplo las desarrolladas por Tall (1981), quien examina cómo la manera en que se introduce a los estudiantes en el estudio de la derivada se ve directamente relacionado con la dificultad de comprensión. Desde su análisis, la introducción de mayor dificultad es la realizada a través de técnicas que involucran definiciones muy sofisticadas, mientras que el uso de los métodos numéricos, en particular, el límite dinámico que se puede manejar a través de representaciones gráficas en las computadoras, parece resultar más adecuado. Este resultado impulsa el análisis de las implicaciones en el aprendizaje de los estudiantes cuando se enseña el cálculo a través de métodos numéricos y de las tecnologías de la información y la comunicación (TIC) (Tall, 1985a, 1985b, 1990a, 1990b, 2009; Tall y Katz, 2014).

Por otra parte, teorías como el enfoque ontosemiótico del conocimiento y la instrucción matemática (EOS) (Godino y Batanero, 1994) han desarrollado investigaciones sobre diferentes fenómenos didácticos relacionados con el aprendizaje y enseñanza de la derivada; se pueden ver por ejemplo los trabajos de Font (2000a, 2000b, 2005, 2009) que abordan aspectos de la derivada como la descripción de sus significados institucionales y personales, su faceta epistémica, acercamientos a su didáctica, análisis y valoración de sus procesos de instrucción, entre otros.

Las investigaciones y aportes sobre este objeto son amplios, además de los trabajos ya mencionados se pueden ver los aportes de Dolores (1998), Baker, Cooley y Trigueros (2000), , Sánchez-Matamoros (2008), Salazar, Díaz y Bautista (2009), entre otros; sin embargo, el constante avance de las TIC ha traído consigo un interés latente por proponer estrategias para su implementación en los cursos de cálculo diferencial (Bedoya y Rico, 1998; Cortés, Guerrero, Morales y Pedroza, 2014; NCTM, 2000; entre otros).

Sin embargo, no basta solo con la implementación de las TIC, pues de ser así se cae en los errores que llevaron a las diversas críticas hechas a estrategias como los bloques lógicos de Zoltan Dienes, las regletas de Cuisenaire, el minicomputador de George Papy, entre otras, las cuales se basaban simplemente en la trivial introducción acrítica de unos instrumentos o, visto de otra forma, en cómo enseñar, idealizando los instrumentos y olvidando que pueden traer consigo también implicaciones negativas (D'Amore y Fandiño, 2015).

A partir de lo expuesto hasta el momento y en búsqueda de una incorporación didáctica de las TIC en la enseñanza del cálculo diferencial, se encontró que la metodología clase invertida (flipped classroom) (Bergmann y Sams, 2012) ha surgido como una opción de bastante aceptación por parte de profesores de diferentes instituciones de formación para la enseñanza de diversas ciencias.

Surge el interrogante que guía la investigación: ¿̇La implementación del aula invertida favorece realmente el aprendizaje de la derivada en estudiantes universitarios?, siendo el objetivo principal analizar las implicaciones 
en el aprendizaje de los estudiantes del concepto de la derivada al implementar el aula invertida, describiendo los principales factores que benefician o no el aprendizaje del concepto de derivada con la incorporación de esta metodología.

Este estudio se realiza con estudiantes de primer semestre de diferentes carreras de una universidad colombiana, quienes participan en dos sesiones de clase de aula invertida en donde se desarrolla el tema aplicaciones de la derivada: cociente incremental (economía), en la física (velocidades) y finalmente en la determinación de valores máximos y mínimos de una función. En el desarrollo de la actividad se analizan las dinámicas, comentarios, actitudes, preguntas, dificultades y demás aspectos que surgieron en el aula; además se tomaron videos desarrollados por los estudiantes para observar la forma en que expresaban sus ideas y si correspondían a una interpretación correcta de la derivada dentro de los problemas que debían abordar.

Con la información obtenida y analizada se procede a discutir los resultados y se da una primera evaluación de la metodología implementada, evaluación que es comparada con los resultados obtenidos por los estudiantes en las pruebas generales de la universidad, esto con la finalidad de dar un punto de vista objetivo y presentar las respectivas conclusiones de la investigación.

\section{Marco teórico}

El desarrollo de estrategias que buscan hacer al estudiante más autónomo en su relación con el conocimiento a través de las TIC, son relativamente recientes. Para hablar particularmente del aula invertida, se debe iniciar por mencionar algunas metodologías que impulsaron su surgimiento como respuesta a la inconformidad de los docentes con la metodología tradicional de clase.

La instrucción entre pares (peer instruction) desarrollada en Estados Unidos por Eric Mazur (1997), en medio de su trabajo como profesor de física de la Universidad de Harvard en los años noventa, marcó un claro punto de partida para el aula invertida; esta propuesta parte de dar a los estudiantes la explicación teórica, ejemplos y demás, que usualmente se dan durante la clase, a través de notas y videos que deben ser estudiadas y hechas propias antes del encuentro con el profesor, buscando que el salón de clase no sea un espacio de transmisión de información unidireccional (profesor al estudiante), sino que sea un lugar de comunicación, debate y análisis de conceptos, ejercicios y problemas.

Crouch y Mazur (2001) explican que una clase bajo esta metodología inicia con la lectura y análisis de las notas de estudio personal o en grupos por parte de los estudiantes (elaboradas y entregadas por el profesor desde el inicio del curso), luego al reunirse en la sesión de clase los estudiantes hacen preguntas y piden aclaraciones; el profesor responde y, si lo considera necesario, hace aclaraciones adicionales en aspectos que considera de mayor dificultad para el estudiante. El segundo momento consiste en un test conceptual, en donde se presenta una 
pregunta para responder de forma individual; una vez el profesor recibe todas las respuestas muestra cuántas personas acertaron (sin decir quiénes y teniendo en cuenta que si más del $70 \%$ no acierta, entonces se debe explicar de nuevo el concepto relacionado). En seguida, se da espacio para que los estudiantes se reúnan en grupos y discutan sobre las respuestas; pasados $4 \circ 5$ minutos se pide nuevamente que contesten a las preguntas que tuvieron respuestas incorrectas, se muestra el número de aciertos y el profesor explica cuál es la respuesta correcta y por qué.

Para la misma época (finales de los noventa), Gregor Novak y algunos colaboradores proponen una estrategia que va en la misma dirección, el Just-in-Time Teaching (JiTT) (Novak, Patterson, Gavrin y Christian, 1999), metodología pensada desde los fundamentos del constructivismo, específicamente en la idea de guiar el aprendizaje del estudiante a partir de sus conocimientos previos (Guertin, Zappe y Kim, 2007), pero involucrando las TIC para el trabajo autónomo del estudiante previo a las sesiones presenciales de clase.

El desarrollo de esta estrategia parte de la creación de un curso virtual a través de páginas web, en donde el estudiante encuentra semanalmente una serie de actividades y cuestionarios que debe solucionar antes de llegar a la clase correspondiente. Una vez en el aula se dialogan, discuten y explican los conceptos que el docente reconoce como complejos para el estudiante (esto se suele hacer a partir de las soluciones encontradas en la página web).

Estas dos estrategias se han visto fuertemente potenciadas con los avances de las TIC y aun hoy en día presentan una fuerte acogida por parte de docentes e investigadores (principalmente de Norteamérica), esto se puede observar en plataformas como el Centro de Recursos para las Ciencias de la Educación que proporciona recursos electrónicos y ejemplos de preguntas JiTT; sin embargo, el surgimiento del aula invertida se ha ganado el interés de gran parte de los docentes que buscan implementar este tipo de metodologías.

El aula invertida es una metodología de clase cuyo nombre proviene de la exploración Inverted Classroom realizada por Lage, Platt y Treglia (2000) en una clase de economía (Talbert, 2012; Tucker, 2012), exploración que fue perfeccionada por Bergmann y Sams (2012) bajo el nombre Flipped Classroom, siendo implementada inicialmente en clases de química y más tarde popularizada en diferentes áreas.

La idea inicial de Bergmann y Sams era ayudar con videos a aquellos estudiantes que faltaban a clase (Coufal, 2014; Talbert, 2014); sin embargo, notaron que podían sacar mayor provecho de estas grabaciones, así que buscaron darle una mayor estructura y llevarlas a todos los estudiantes, popularizando sus lecciones y dando vida a la organización The Flipped Learning Network, en la cual se reconoce la clase invertida como una estrategia que transfiere el trabajo de algunos procesos de aprendizaje fuera del aula, dando el tiempo de clase para que el profesor pueda facilitar y potenciar otros aspectos relacionados con el aprendizaje de los estudiantes (Bergman y Sams, 2012).

En esta metodología el docente no llega al salón a dar clase, pues ya la grabó previamente para que sus estudiantes la vieran antes en casa o en el lugar que estuvieran, convirtiéndose el profesor en un tutor y apoyo más personalizado (Bergmann y Sams, 2012). El tiempo de clase presencial es utilizado por el profesor para abordar ejercicios y problemas que inviten al estudiante a poner a prueba su comprensión de los conceptos, dando espacio para el trabajo en grupo y también para el debate y las inquietudes de los estudiantes. 
Entre los diferentes modelos y teorías en la educación, se puede decir que, de acuerdo con la taxonomía de Staker y Horn (2012), el aula invertida encaja en los submodelos de rotación o entornos mixtos, al ser una metodología en la cual el estudiante aprende a través de recursos en línea, controlando de manera significativa aspectos como el tiempo, el lugar de estudio, la dedicación o el esfuerzo, pero también debe desarrollar parte de su proceso de aprendizaje de manera presencial en el salón de clases (Christensen, Hor y Staker, 2013), entrelazamiento que ha sido denominado como blended learning.

Además, guarda relación con el sustento teórico del constructivismo de Vygotsky (Davies, Dean y Ball, 2013), al ofrecer espacios de trabajo colaborativo para la resolución de problemas y también con la teoría del aprendizaje experiencial expuesta por Kolb y Yeganeh (2009), en la cual el estudiante reflexiona continuamente sobre lo que experimenta para poder construir conceptos (Coufal, 2014).

Bergmann y Sams (2012) proponen el desarrollo del modelo de aula invertida bajo las siguientes etapas:

1. Creación de un sitio web de fácil acceso para el estudiante, en donde se dispondrán los diferentes videos y recursos del curso, resaltando la importancia de la presencia de chats y foros.

2. Comunicación a los estudiantes y padres de familia (esto en caso de trabajar con menores de edad) de la estrategia que se utilizará, en qué consiste, qué temas se abordarán. Además, se escuchan sus opiniones sobre lo que se hará.

3. Sesión de adaptación a la plataforma o recursos virtuales, dando a conocer la forma de acceso, revisando que todos los estudiantes puedan acceder a los contenidos y dando consejos a los estudiantes de cómo puede ser el tiempo de estudio independiente.

4. Para el trabajo en las clases presenciales posteriores, los estudiantes deben preparar al menos una pregunta sobre la temática expuesta en los videos preparados por el docente, la cual no puede ser resuelta directamente con el video (esto en búsqueda de posibles aspectos que no quedaron claros o no se abordaron en la explicación del docente).

5. Se desarrollan actividades en grupo, en las cuales el estudiante puede recurrir al uso de internet, computadores, celulares, libros, etc.

6. Se debe realizar una evaluación constante, que le dé al estudiante una retroalimentación para la reflexión de su proceso y estudio de los aspectos débiles en su proceso de aprendizaje.

Estos aspectos teóricos del aula invertida despiertan gran interés y motivación por su aplicación; sin embargo, es importante analizarla en su puesta en práctica en las instituciones educativas en busca de resaltar sus aportes y las posibles desventajas en contextos específicos, ya que, idealizar una metodología no es lo más correcto. 
Por otra parte, y teniendo claro que el objetivo de este trabajo no es hacer un profundo análisis sobre las diferentes corrientes y planteamientos del constructivismo en la educación, se toma como posición para la discusión de los resultados encontrados, el referirse a la construcción del conocimiento tomando como base las concepciones del constructivismo radical, específicamente, los planteamientos de Glasersfeld (1990, 1991, 2007), quien expone que:

Llamamos a esta escuela del constructivismo "radical" porque sostiene que la actividad perceptual (y conceptual) del conocedor no es meramente la de seleccionar o transformar estructuras cognitivas por medio de alguna forma de interacción con estructuras "existentes", sino más bien una actividad constitutiva que, solo, es responsable de cada tipo o tipo de estructura que un organismo llega a "saber". Y esto nos lleva al segundo punto epistemológicamente importante en el análisis de la permanencia del objeto. (Glasersfeld, 2007, p. 78)

Sosteniendo dos supuestos primordiales:

1. El conocimiento no se recibe pasivamente, sino que se desarrolla activamente por el sujeto cognitivo.

2. La función de la cognición es adaptativa y sirve a la organización del mundo experiencial, no al descubrimiento de la realidad ontológica (Glasersfeld, 1991, p. 31).

\section{Metodología}

En este trabajo se implementó el aula invertida en el curso de cálculo diferencial conformado por 25 estudiantes de primer semestre de una universidad colombiana; estos estudiantes pertenecen a programas de ingeniería y administración de empresas. La experiencia se desarrolló en dos sesiones de clase, cada una de dos horas, y la temática abordada (aplicaciones de la derivada) corresponde a la parte final del curso.

Al generar una propuesta para la enseñanza y aprendizaje de un concepto se corre el riesgo de caer en un simple tecnicismo en el que se puede desconocer la esencia del fenómeno estudiado; por esta razón, en este trabajo se buscó una interpretación y comprensión más cercana al problema planteado, mediante la revelación de mecanismos y significados ocultos que se dan en él (Fiorentini y Lorenzato, 2010), para ello se siguieron las características básicas de la investigación cualitativa. Se utiliza el entorno natural como fuente directa de los datos y el investigador como su instrumento principal. Los datos recogidos son predominantemente descriptivos. La preocupación por el proceso es mucho mayor que el producto. El "significado" que da la persona de las cosas y su vida son foco de atención especialmente por el investigador. El análisis de los datos tiende a seguir un proceso inductivo (Lüdke y Andrew, 1986).

Por lo anterior, se desarrollaron las siguientes etapas:

1. Delimitación: a partir de los resultados en las pruebas institucionales del curso de cálculo diferencial en semestres académicos inmediatamente precedentes, se constató que los estudiantes presentan dificultades para aplicar el concepto de derivada en la resolución de ejercicios de aplicación.

2. Diseño: se desarrollaron dos videos de explicación por parte del profesor de la relación de la derivada con situaciones de: cociente incremental (economía), la física (velocidades) y valores máximos y mínimos. Estos 
videos se dejaron a disposición de los estudiantes a través de la plataforma de YouTube. Para la creación de estos videos el profesor tomó los datos de la variación del precio del barril de petróleo para Colombia durante tres meses, presentando un análisis gráfico de la información y la correspondiente determinación de cocientes incrementales y cómo se relacionaban estos con el concepto de derivada. En cuanto al segundo video el profesor se ubica en una de los parqueaderos de las instalaciones de la universidad y registra datos relacionados con el desplazamiento, tiempo y velocidad con la que ingresan algunos estudiantes en bicicleta, con lo cual presenta un gráfico del desplazamiento en función del tiempo y hace uso de la derivación para contrastar con las velocidades. Además, como trabajo en el aula los estudiantes debían abordar alguna de las siguientes situaciones:

- Variación en los principales indicadores económicos de Colombia. Para este tema cada estudiante eligió un indicador (producto interno bruto, deuda externa, exportaciones, importaciones, etc.) y a partir de los datos que se encuentran en la página del Banco de la República de Colombia, debían analizar la razón de cambio del indicador para ciertos años.

- La velocidad con la cual circulan los vehículos en la vía principal de acceso a la universidad. Para esta actividad se asignó un tramo de la vía a cada grupo de estudiantes (tres estudiantes por grupo), en el cual debían registrar desplazamientos y tiempos de los autos, con el fin de que analizaran su razón de cambio y luego explicaran las posibles formas de encontrar esa razón a través de la derivación.

3. Aplicación: para la primera sesión de clase, los estudiantes observaron los videos de explicación y seleccionaron alguna de las situaciones propuestas por el docente. Una vez reunidos en el salón de clase, se dio espacio para preguntas y sus respectivas aclaraciones, ya fuera sobre los aspectos matemáticos o la situación seleccionada. Cada grupo de estudiantes inició la toma de datos y el análisis correspondiente. Para la segunda sesión, los estudiantes presentaron los resultados de su ejercicio, dejando a disposición de sus compañeros y del profesor los videos de cómo se realizaron todos los procesos. Finalmente, se abrió un espacio de diálogo para conocer la opinión de los estudiantes sobre lo realizado y el docente dio a conocer los resultados de la actividad.

4. Análisis: se organizó, sistematizó y se trató de dar la mejor interpretación de la información recogida en la etapa de aplicación. Para ello se grabaron las dos sesiones de clase, se realizó un diario de campo en el cual se anotaron las percepciones inmediatas del docente, luego se organizaron y analizaron los videos realizados por los estudiantes. 


\section{Resultados y discusión}

\section{Aspectos conceptuales}

El manejo del concepto matemático perseguido en la actividad es fundamental, por esta razón se presta particular atención al tipo de inquietudes que surgieron de los estudiantes en la primera sesión de clase, en donde se revela la dificultad que representa la idea de derivada como un límite, ya que, el carácter dinámico del cálculo no es fácil de captar en explicaciones teóricas, pues el carácter dinámico que se pretende dar a través de un software llega a ser limitado lo cual difiere por completo del concepto de derivada, lo anterior se evidenció en preguntas de los estudiantes como:

- Profesor, żla suma de las secantes forma la tangente?

- Si las secantes tocan la función en dos partes, ¿̇cómo pueden volverse una tangente?

- ¿Cómo puede ser la diferencia de coordenadas cero? ¿Ya no tendríamos dos puntos para la razón?

Ante estas inquietudes se hace necesaria una intervención adicional del profesor (no planeada previamente), quien da algunas ideas de los aspectos dinámicos involucrados y abre un diálogo grupal, escuchando y confrontando las ideas de los estudiantes, en búsqueda de unificar ideas y criterios para el análisis del papel del límite de la función en la aproximación a las rectas tangentes.

Además, el hecho de abordar una situación concreta a través de un concepto matemático sin recurrir a los algoritmos usuales de derivación genera en el estudiante inquietud y una desorientación inicial, siendo evidencia del poco manejo de las diferentes concepciones de la derivada que se alcanza con un modelo de explicación teórica tradicional.
Esto se demuestra en los videos realizados por los estudiantes, en los que hacen uso de expresiones como:

- Al no tener una función para derivar żnos toca usar el límite?

- Como tenemos puntos y no funciones ¿debemos usar el límite?

- $\quad$ ¿El límite nos indica qué tan rápido cambiaron las importaciones?

Expresiones en las cuales el estudiante intenta argumentar el uso del algoritmo sin relacionar la expresión utilizada con la definición de derivada; este aspecto fue puesto en discusión en el tiempo final de socialización de las actividades, lográndose relacionar los conceptos mediante el análisis de los resultados de los grupos de trabajo; es decir, los estudiantes lograron relacionar los conceptos de límite, cociente incremental, velocidades y derivada sin que el docente explicitara estas relaciones.

Otro de los aspectos que se evidencia en los videos realizados por los estudiantes es que todos lograron reconocer que en los puntos máximos y mínimos el límite tiende a cero; sin embargo, en el desarrollo de su argumentación no evidencian el haber identificado la correspondiente relación de este límite con la pendiente de la recta tangente; razón por la cual el profesor lleva este aspecto a discusión justo en el momento posterior al reconocimiento de la relación entre los límites calculados y la derivada, para ello interviene diciendo:

- Si los límites que calcularon dan cero en puntos máximos y mínimos żse puede decir algo en particular de la derivada en estos puntos?

Ante esto los estudiantes dan diferentes argumentos que de manera progresiva los llevan a detectar que la pendiente de la recta tangente en estos puntos es cero, es decir, la recta es paralela al eje de la variable independiente en el plano cartesiano. 


\section{Aspectos del aula invertida}

Esta implementación permitió analizar las principales características de esta metodología, encontrando aspectos tanto positivos como negativos. En cuanto a los aspectos positivos se pude destacar lo siguiente.

En la actividad desarrollada los estudiantes participaron en su totalidad en la entrega del producto solicitado (video), en el cual recurrieron a diferentes elementos creativos con los que se llegó a un nivel de calidad de diseño superior a la propuesta por el profesor. Esto y la dinámica con la cual los grupos desarrollaron la actividad, son muestra de que se logró motivar a los estudiantes por ser parte del trabajo de la asignatura, siendo fundamental la inclusión que se hizo de la tecnología y, en especial, las redes sociales que usualmente utilizan y que poco ven involucradas en las clases de cálculo.

En los diferentes grupos de trabajo se encontró, como era de esperarse, que algunos estudiantes tenían una mayor comprensión de lo propuesto; por esta razón, se encuentra en las grabaciones de clase un comportamiento de guía o monitor por parte de ellos, recurriendo al profesor únicamente para aclaración de aspectos específicos que les permitiera explicar de mejor forma sus ideas a los compañeros de grupo. Este aspecto le permitió al profesor acercarse y acompañar más a los estudiantes que tenían dificultades en su aprendizaje.

Se logró generar espacios de trabajo grupal significativos, donde los estudiantes confrontaron ideas y procedimientos para unificar criterios y superar dificultades, pues en el momento de socializar e institucionalizar conceptos, los estudiantes sentían la responsabilidad, e incluso el reto, de expresar sus ideas, argumentarlas y, de ser necesario, replantearlas, de forma que los conceptos e ideas que el profesor daba por acertadas resultaban ser una construcción de la clase y no algo ajeno al estudiante.

Las pruebas generales de la universidad desarrolladas después de la implementación del aula invertida permitieron analizar el comportamiento de los estudiantes ante problemas relacionados con el manejo del concepto de derivada en situaciones de cociente incremental, razón de cambio y optimización de funciones, encontrando que la mayoría de ellos alcanzaron un buen dominio de conceptos y algoritmos. Se destaca en estas pruebas una mayor capacidad de argumentación en los procesos desarrollados por parte de los estudiantes con respecto a las pruebas estudiadas en la etapa de delimitación.

Tratar de explicar las razones por las cuales optaban por un procedimiento y explicitar por qué era oportuno el uso de los algoritmos de la derivada en las soluciones de los ejercicios, fue un aspecto recurrente y distintivo encontrado en las pruebas desarrolladas por los estudiantes que se observaron en este trabajo. Las figuras 1, 2 y 3 son muestra de lo anterior. 
Lo tratado hasta el momento expone aspectos que justifican los beneficios del aula invertida; sin embargo, en la implementación y análisis, también se evidencian aspectos negativos. A continuación se hablará sobre ellos.

Un punto que claramente sale a relucir en esta metodología de clase es que el trabajo depende en gran parte de la colaboración de los estudiantes, pero żqué pasa si los estudiantes no tienen esa disposición? ¿̇O si en el núcleo familiar, y a pesar de una aceptación previa, no se está de acuerdo con ella? En esta experiencia, en particular, se pidió la aprobación de todos los estudiantes; sin embargo, uno de ellos decidió no participar en el trabajo de aula, pues manifestó su preferencia por el método tradicional de clase.

Ahora, no todos los aspectos relacionados con el aprendizaje de un objeto matemático son trabajados de manera significativa bajo esta metodología; por ejemplo, en el trabajo algorítmico se cae en el tradicionalismo en el cual simplemente se da información, es decir, el estudiante puede observar una serie de ejemplos y alcanzar un manejo de los diferentes procedimientos, reglas y propiedades, pero esto no es garantía de que el estudiante comprenda realmente lo que está detrás de ellos, pues no son resultado de un proceso cognitivo propio.

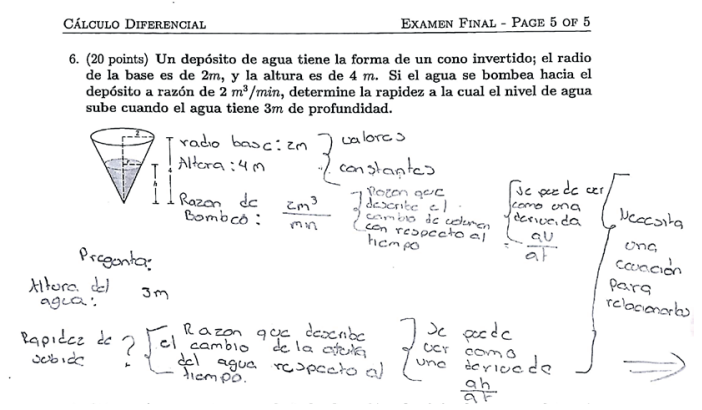

Figura 1. Argumentación de estudiante en la solución de ejercicio relacionado con la derivada como razón de cambio

Fuente: elaboración propia.
Esta metodología limita el desarrollo de clase de modo constructivista, ya que, al obtener los estudiantes la información previa a la clase, pueden buscar en otros medios la información que consideren necesaria, adquiriendo productos terminados que luego llevan a las actividades de aula, con lo cual el estudiante no desarrolla un proceso para crear una idea propia del objeto matemático. Además, al dar la información a través de videos, se asume que el mensaje que pretende dar el profesor es captado por el estudiante de la manera esperada, dejando a un lado que "los conceptos y el nuevo conocimiento no pueden transmitirse simplemente a otra persona al hablarle, porque cada uno debe abstraer significados, conceptos y conocimientos de su propia experiencia" (Glasersfeld, 1991, p. xiv, sección de introducción).

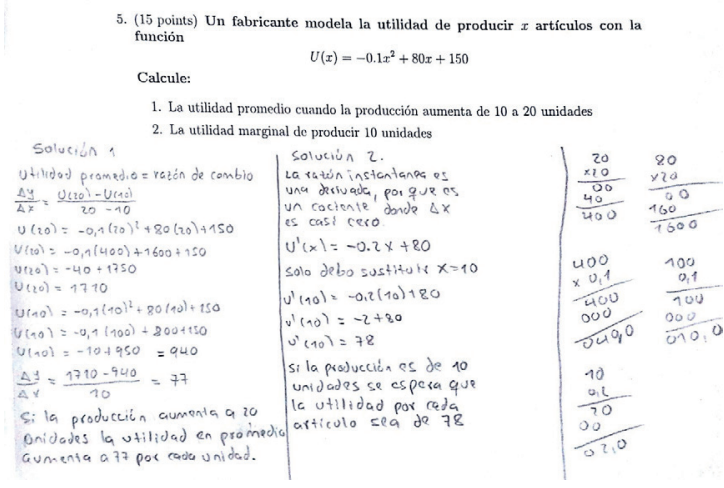

Figura 2. Solución por parte de estudiante a ejercicio relacionado con la derivada como cociente incremental

Fuente: elaboración propia. 


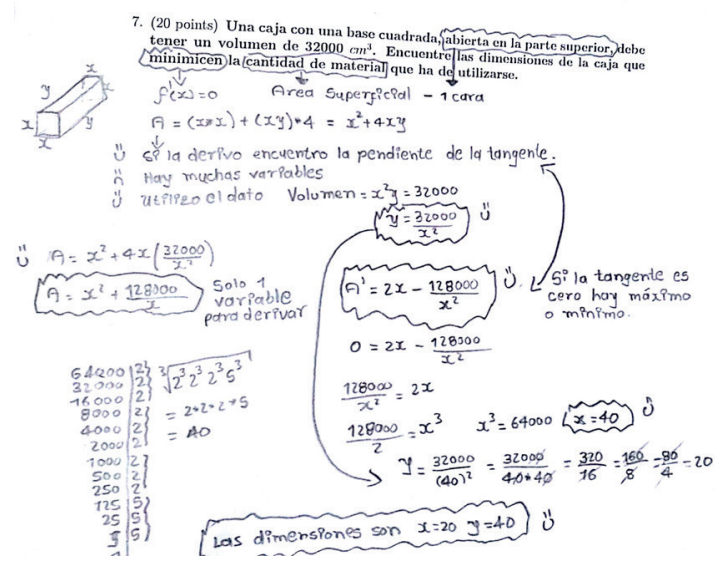

Figura 3. Solución por parte de estudiante a ejercicio relacionado con optimización de una función.

Fuente: elaboración propia.

La libertad de los estudiantes para recurrir a diversas fuentes de información puede verse reflejada negativamente, pues no hay garantías de que las fuentes a las que recurren sean confiables; es decir, están expuestos a la generación de futuros conflictos semióticos y cognitivos. Un ejemplo de lo anterior es la recurrente expresión "reemplazamos la variable x por infinito" que se utiliza en tutoriales de video para el análisis de límites de funciones cuando la variable dependiente tiende a infinito, creando en el estudiante la idea del infinito como un número.

Demanda gran cantidad de tiempo por parte del profesor para la preparación de clase, este aspecto se toma como negativo considerando las condiciones de muchos docentes en diferentes instituciones de educación formal en determinados países, en donde deben manejar grupos considerablemente numerosos y además de diferentes asignaturas; por lo cual, pensar en dedicar un gran número de horas a la preparación de una sola sesión de clase se vuelve inviable. Lo anterior teniendo en cuenta que una correcta implementación del aula invertida no considera utilizar los mismos videos explicativos del docente una y otra vez en los diferentes grupos de clase, ya que, se busca explicar al estudiante con la ayuda de videos que le resulten interesantes y este "interés" puede variar en cada grupo de clase.

A pesar de los aspectos negativos que se evidenciaron bajo el manejo de esta metodología, la experiencia resultó positiva tanto para los estudiantes como para el docente, en vista de que se logró el objetivo de que los estudiantes manejaran la aplicación del concepto de la derivada en la resolución de ejercicios de aplicación. 


\section{Conclusiones}

Lo anterior puede indicar que esta metodología sirve como alternativa para romper con las rutinas de clase y que puede ser utilizada ocasionalmente para motivar a los estudiantes o para desarrollar temáticas que el docente considere propicias. Sin embargo, es claro que este modelo presenta aún algunas limitaciones pedagógicas al obviar procesos fundamentales en el aprendizaje de la matemática como, por ejemplo, la personalización, significación, idealización y representación, estos procesos son definidos y explicados por Font y Rubio (2017). Además, de cierto modo dificulta el aprendizaje a través de la construcción de conocimiento, ya que no parte de los conocimientos previos del estudiante si no que es el docente quien da la información inicial de conceptos y demás.

Otro aspecto importante que se encontró en esta experiencia es que bajo la metodología de aula invertida las TIC toman un papel de instrumento transmisor, ya que se hace uso de redes sociales y herramientas con el único objetivo de elaborar una clase en la cual se da al estudiante una información y no hay mayores implicaciones de carácter pedagógico en este papel que asumen.

Un posible contexto de aplicación más propicio para esta metodología es la educación a distancia, la cual ocupa un espacio significativo hoy en día en el sector educativo de algunos países, ya que los elementos presentes en esta metodología pueden enriquecer la forma en que se da la comunicación entre docentes y estudiantes.

Finalmente, se puede decir que, si bien esta nueva tendencia en la enseñanza puede resultar útil en diferentes aspectos, aún debe ser pensada con mayor profundidad de forma que atienda realmente a las necesidades y dificultades que enfrentan los estudiantes en sus procesos de aprendizaje, pues hasta el momento se hace mayor énfasis en cómo enseñar y no en cómo aprender.

\section{Referencias}

Baker, B., Cooley, L. y Trigueros, M. (2000). A calculus graphing schema. The Journal for Research in Mathematics Education, 37 (5), 557-578. doi: 10.2307/749887

Bedoya, E. y Rico, L. (1998). Calculadoras graficadoras y enseñanza de matemáticas en secundaria. En Actas del iv Simposio sobre Investigación en el Aula de Matemáticas (pp. 113-131). Granada: Thales.

Bergmann, J. y Sams, A. (2012). Flip your classroom: Reach every student in every class every day. Eugene: International Society for Technology in Education.

Brousseau, G. (1986). Fondements et méthodes de la didactique des mathématiques. Recherches en Didactique des Mathématiques, 7(2), 33- 115.

Cortés, C., Guerrero, L., Morales Ch. y Pedroza, L. (2014). Tecnologías de la información y la comunicación (TIC). Aplicaciones tecnológicas para el aprendizaje de las matemáticas. Unión: Revista Iberoamericana de Educación Matemática, 39, 141-161.

Coufal, K. (2014). Flipped learning instructional model: perceptions of video delivery to support engagement in eighth grade math. (Tesis doctoral, Lamar University). De la base de datos ProQuest Dissertations Publishing. (UMI No. 3634205)

Christensen, C., Horn, M. y Staker, H. (2013). Is K-12 blended learning disruptive: An introduction of the theory of hybrids. Recuperado del sitio de Internet del Clayton Christensen Institute. San Francisco, Estados Unidos. http://www.christenseninstitute.org/ wp-content/uploads/2013/05/Is-K-12-Blended-Learning-Disruptive.pdf 
Crouch, C. y Mazur, E. (2001). Peer instruction: Ten years of experience and results. American Journal of Physics, 69(9), 970-977. Recuperado de http://aapt.scitation. org/doi/citedby/10.1119/1.1374249

D’Amore, B. y Fandiño, M. (2015). Propuestas metodológicas que constituyeron ilusiones en el proceso de enseñanza de la matemática. Educación Matemática, 27(3), 7-43. Recuperado de http://www.redalyc.org/articulo.oa? id=40544202001

Davies, R., Dean, D. y Ball, N. (2013). Flipping the classroom and instructional technology integration in a college-level information systems spreadsheet course. Educational Technology Research and Development, 61 (4), 563-580.

Dolores, C. (1998). Algunas ideas que acerca de la derivada se forman los estudiantes del bachillerato en sus cursos de cálculo diferencial. En F. Hitt (ed.), Investigaciones en matemáticas educativas ii, 257-272. México: Grupo Editorial Iberoamérica.

Fiorentini, D. y Lorenzato, S. (2010). Investigación en educación matemática: recorridos históricos y metodológicos (Trad. A. Jiménez). Campinas: Autores Associados.

Font, V. (2000a). Procediments per obtenir expressions simbóliques a partir de gráfiques. Aplicacions a les derivades. (Tesis doctoral). Universitat de Barcelona, España.

Font, V. (2000b). Representaciones ostensivas que pueden ser activadas en el cálculo de $f^{\prime}(x)$. El caso de la función seno. Uno: Revista de Didáctica de las Matemáticas (25), $21-40$.

Font, V. (2005). Una aproximación ontosemiótica a la didáctica de la derivada. En A. Maz, B. Gómez y M. Torralbo (eds.), Investigación en educación matemática. Noveno Simposio de la Sociedad Española de Investigación en Educación Matemática, 109-128. Córdoba, Universidad de Córdoba.

Font, V. (2009). Formas de argumentación en el cálculo de la función derivada de la función $f(x)=x^{2}$ sin usar la definición por límites. Unión: Revista Iberoamericana de Educación Matemática (18), 15-28.

Font, V. y Rubio, N. V. (2017). Procesos matemáticos en el enfoque ontosemiótico. En J. M. Contreras, P. Arteaga, G. R. Cañadas, M. M. Gea, B. Giacomone y M. M. López-Martín (eds.), Actas del Segundo Congreso International Virtual sobre el Enfoque Ontosemiótico del Conocimiento y la Instrucción Matemáticos. Recuperado de https://www.researchgate.net/publication/314195166_Procesos_matematicos_en_el_enfoque_ontosemiotico

Glasersfeld, E. (1990). An exposition of constructivism: Why some like it radical. En R. Davis, C. Maher, y N. Noddings (eds.), Constructivist views on the teaching and learning of mathematics (pp. 19-29). Reston: National Council of Teachers of Mathematics (NCTM).

Glasersfeld, E. (1991). Constructivism in education. En A. Lewy (ed.), The international encyclopedia of curriculum. Oxford: Pergamon Press. 
Glasersfeld, E. (2007). Key works in radical constructivism. Rotterdam: Sense Publishers.

Godino, J. y Batanero, C. (1994). Significado institucional y personal de los objetos matemáticos. Recherches en Didactique des Mathématiques, 14(3), 325-355.

Godino, J., Batanero, C. y Font, V. (2007). The ontosemiotic approach to research in mathematics education. $z d m$ : The International Journal on Mathematics Education, 39(1-2), 127-135.

Guertin, L., Zappe, S. y Kim, H. (2007). Just-intime teaching exercises to engage students in an introductory-level dinosaur course. Journal of Science Education and Technology, 16(6), 507-514.

Kolb, D. y Yeganeh, B. (2009). Mindfulness and experiential learning. od Practitioner, 41 (3), 13-18.

Lage, M., Platt, G. y Treglia, M. (2000). Inverting the classroom: A gateway to creating an inclusive learning environment. The Journal of Economic Education, 37 (1), 30-43.

Lüdke, M. y André, M. (1986). Pesquisa em educação: abordagens qualitativas. São Paulo: Editora Pedagógica e Universitária.

Mazur, E. (1997). Peer instruction. A user's manual. New Jersey: Prentice Hall, Inc.

National Council of Teachers of Mathematics. (2000). Principles and standards for school mathematics. Reston: Autor.

Novak, G, Patterson, E., Gavrin, A. y Christian, W. (1999). Just-in-time teaching: Blending active learning with web technology. Upper Saddle River, New Jersey: Prentice Hall.

Salazar, C., Díaz, H. y Bautista, M. (2009). Descripción de niveles de comprensión del concepto derivada. Tecné, Episteme y Didaxis (26), 62-82. Recuperado de http://www.pedagogica.edu.co/admin/UserFiles/12(1).pdf
Sánchez-Matamoros, G. (2004). Análisis de la comprensión en los alumnos de bachillerato y primer año de la universidad sobre la noción matemática de derivada (desarrollo del concepto). (Tesis de doctorado) Universidad de Sevilla, Sevilla. Recuperada de hittps://dialnet. unirioja.es/servlet/tesis? codigo $=59750$.

Staker, H. y Horn, M. (2012). Classifying K-12 blended learning. Clayton Christensen Institute. San Francisco, Estados Unidos. Recuperado de https://www.christenseninstitute.org/wp-content/uploads/2013/04/ Classifying-K-12-blended-learning.pdf

Talbert, R. (2012). Inverted classroom. Colleagues, 9(1), 1-2. Recuperado de http://scholarworks.gvsu.edu/colleagues/vol9/iss $1 / 7$

Talbert, R. (2014). Inverting the linear algebra classroom. Primus: Problems, Resources, and Issues in Mathematics Undergraduate Studies, 24(5), 361-374. Recuperado de http://www.tandfonline.com/doi/pdf/10.10 $80 / 10511970.2014 .883457$

Tall, D. (1981). Comments on the difficulty and validity of various approaches to the calculus. For the Learning of Mathematics, 2(2), 16-21. Recuperado de https://www. jstor.org/stable/40247733? $\mathrm{seq}=1$ \# page_scan_tab_contents

Tall, D. (1985a). Understanding the calculus. Mathematics Teaching, 110, 49-53. Recuperado de http://wrap.warwick.ac.uk/495/

Tall, D. (1985b). Tangents and the Leibniz notation. Mathematics Teaching, 1 12, 48-52. Recuperado de http://homepages.warwick. ac.uk/staff/David.Tall/pdfs/dot1985d-tgtleibniz-mt.pdf

Tall, D. (1990a). Inconsistencies in the learning of calculus and analysis. Focus on Learning Problems in Mathematics, 12(3), 49-63. Recuperado de http://homepages.warwick. ac.uk/staff/David.Tall/pdfs/dot1990b-inconsist-focus.pdf 
Tall, D. (1990b). A versatile approach to calculus and numerical methods. Teaching Mathematics and its Applications, 9(3), 124-131.

Tall, D. (2009). Dynamic mathematics and the blending of knowledge structures in the calculus. zdm: The International Journal on Mathematics Education, 41 (4), $481-492$.

Tall, D. y Katz, M. (2014). A cognitive analysis of cauchy's conceptions of function, continuity, limit, and infinitesimal, with implications for teaching the calculus. Educational Studies in Mathematics, 86(1), 97-124.

Tucker, B. (2012). The flipped classroom. Education Next, 12(1), 82-83.

\section{Para citar este artículo}

Fúneme, C. (2019). Aplicaciones de la derivada a través del aula invertida. Tecné, Episteme y Didaxis: TED, 45, 159-174. 